\title{
Ascertaining Shearer Design Plan Based on Quantization of Weight of Multiple Attributes
}

\author{
Fan Qiuxia and Yang Zhaojian* \\ College of Mechanical Engineering, Taiyuan University of Technology, Taiyuan, China
}

\begin{abstract}
To eliminate the instability in deciding upon shearer design plans and take into full account of relevancy among shearers' attributes, the authors propose a way to ascertain the weight of multiple attributes on the basis of sensitivity analysis. With this method, one can work out a matrix through 3-level quantization criteria to compare attributes and then sort design plans accordingly; with interrelationship constraint of multiple attributes, one can obtain the variation range of weight of these attributes that has no impact on the ranking of design plans and identify the significant attribute so as to reach the optimal plan. This method has been proven feasible and practical through sorting the overall performance of design plans of the whole shearer and 6 torque-axis unload grooves in the cutting unit of a shearer.
\end{abstract}

Keywords: Shearer design plan, Weight of attributes, Sensitivity analysis.

\section{INTRODUCTION}

The selection of design plan of a shear has impact on its design \& development cycle and its market performance. Factors involved in selecting a design plan of a shear include weight and values of attributes, such as thickness of coal seam, maximum coal-cutting height, traction and cutting power [1-3]. Domestic and foreign experts have made great efforts on the attributes involved in shearer design [4-6] and yet, there is no special account in related literature about relevance among attributes, which has significant impact on the selection of shearer design plans. Therefore the authors categorize multiple attributes of shearers into independent attributes and relevant ones, and analyze and identify the significant attributes under constraint conditions, and in this way aid designers to obtain more reasonable shearer design plans.

\section{SENSITIVITY ANALYSIS ON WEIGHT OF MUL- TIPLE ATTRIBUTES OF A SHEARER}

Measuring the relative significance of various indicators of a shearer depends largely on the weight of multiple attributes and has impact on the selection of shearer design plan. That the structure and working conditions of a shearer and the ideas of design experts tend to change as time goes, resulting in the change of the significance of each attribute and altering the rank of design results. Thus, it is necessary to quantize multiple attributes to determine the shearer design plan.

\subsection{Ascertaining the Weight of Multiple Attributes of Shearer}

When it comes to ascertain the weight of attributes, subject method and objective method $[7,8]$ are used. Given the

*Address correspondence to this author at the Yingze street, Taiyuan, China, Postcard: 030024; Tel:13834511099; E-mail: yangzhaojian@tyut.edu.cn vital importance of experts' experience and expertise in designing a shearer, it is imperative to bring them in when determining the weight of attributes. Now that there are differences in experts' research fields and their understanding over attributes, the authors of this paper compare the priority of attributes through 3-level quantitative criteria and build up a comparison matrix of attributes. In case attribute $q_{1}$ is more important than attribute $q_{2}$, then $e_{12}=1$ and $e_{21}=-1$. Take a shearer's attributes for example. For reliability $q_{k}$ and traction velocity $q_{q}$, in case $q_{k} \succ q_{q}$, then $e_{k q}=1, e_{q k}=-1$. If attribute $q_{1}$ is as important as $q_{2}$, then $e_{12}=e_{21}=0$ [9].

The procedure to ascertain a shearer's multiple attributes is as follows:

Step 1: Get the comparison matrix E based on the method mentioned above:

$E=\left[\begin{array}{cccc}e_{11} & e_{12} & \ldots & e_{1 n} \\ e_{21} & e_{22} & \cdots & e_{2 n} \\ \vdots & \vdots & \ddots & \vdots \\ e_{n 1} & e_{n 2} & \cdots & e_{n n}\end{array}\right]$

Step 2: Construct transfer matrix $\mathrm{S}$ for $\mathrm{E}$ according to the theory of optimal transfer matrix:

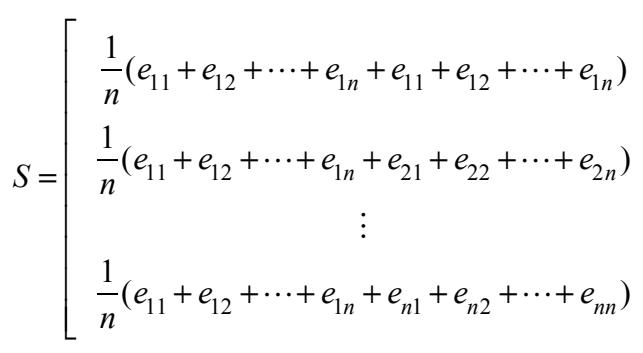




$$
\left.\begin{array}{cc}
\frac{1}{n}\left(e_{11}+e_{12}+\cdots+e_{1 n}+e_{12}+e_{22}+\cdots+e_{n 2}\right) & \cdots \\
\frac{1}{n}\left(e_{12}+e_{22}+\cdots+e_{n 2}+e_{12}+e_{22}+\cdots+e_{n 2}\right) & \cdots \\
\vdots & \ddots \\
\frac{1}{n}\left(e_{n 2}+e_{n 2}+\cdots+e_{n n}+e_{1 n}+e_{2 n}+\cdots+e_{n n}\right. & \\
\frac{1}{n}\left(e_{11}+e_{12}+\cdots+e_{1 n}+e_{12}+e_{22}+\cdots+e_{n n}\right) \\
\frac{1}{n}\left(e_{21}+e_{22}+\cdots+e_{2 n}+e_{12}+e_{22}+\cdots+e_{n n}\right) \\
\vdots \\
\frac{1}{n}\left(e_{n 1}+e_{n 2}+\cdots+e_{n n}+e_{1 n}+e_{2 n}+\cdots+e_{n n}\right.
\end{array}\right]
$$

From which judgment matrix $C=\left(c_{i j}\right)_{n \times n}$ is obtained, in which

$c_{i j}=\exp \left(s_{i j}\right)$

$s_{i j}=\frac{1}{n} \sum_{k=1}^{n}\left(e_{i k}+e_{k j}\right)$

Step 3: calculate weighted values [10]

$$
\omega_{j}=\omega_{j}^{*} \sum_{j=1}^{n} \omega_{j}^{*},
$$

In which $\omega_{j}^{*}=\sum_{i=1}^{n} c_{j i}, j=1,2 \cdots n, \sum_{j=1}^{n} \omega_{j}=1$.

\subsection{Ascertaining Evaluation Values of Shearer Design Plans}

Assume: there are $\mathrm{m}$ shearer design plans available, represented as $p_{1}, p_{2}, \ldots p_{m}$, and $\mathrm{n}$ evaluation indicators, including thickness of coal seam, maximum coal-cutting height, traction and cutting power, etc. represented as $q_{1}, q_{2}, \ldots q_{n}$, and decision matrix of attribute evaluation value $a_{i j}$ :

$$
A=\left[\begin{array}{cccc}
a_{11} & a_{12} & \cdots & a_{1 n} \\
a_{21} & a_{22} & \cdots & a_{2 n} \\
\vdots & \vdots & \ddots & \vdots \\
a_{m 1} & a_{m 2} & \cdots & a_{m n}
\end{array}\right]
$$

Since there are discrepancies in the connotation, dimension and order of magnitude of shearer attributes. For example, two attributes of a common shearer like its cutting power which is usually $100 \sim 1000 \mathrm{~kW}$ and its cutting height which is $1 \sim 10 \mathrm{~m}$ in most cases, vary greatly from one another. To eliminate such discrepancy, the authors here normalize matrix A with membership in fuzzy math's and build up membership matrix R:

$$
\begin{aligned}
& R=\left[\begin{array}{cccc}
r_{11} & r_{12} & \cdots & r_{1 n} \\
r_{21} & r_{22} & \cdots & r_{2 n} \\
\vdots & \vdots & \ddots & \vdots \\
r_{m 1} & r_{m 2} & \cdots & r_{m n}
\end{array}\right] \\
& \text { In which, } r_{i j}=\frac{a_{i j}-\min _{j} a_{i j}}{\max _{j} a_{i j}-\min _{j} a_{i j}} \text { (values of } r_{i j} \text { are in direct } \\
& \text { proportion to attribute sensibility) or } r_{i j}=\frac{\max _{j} a_{i j}-a_{i j}}{\max _{j} a_{i j}-\min _{j} a_{i j}}
\end{aligned}
$$

(values of $r_{i j}$ are in indirect proportion to attribute sensibility).

The comprehensive evaluation values of each design plan are $M_{i}=\sum_{j=1}^{n} \omega_{j} \bullet r_{i j}, i=1,2 \cdots m$. Then, the priority order $p_{1}^{\prime}$, $p_{2}^{\prime}, \ldots p_{m}^{\prime}$ of these design plans can be determined according to these values.

\subsection{Sensitivity Analysis on Weight of Multiple Attributes}

Given the multiple attributes involved in shearer design, such as thickness of coal seam, maximum coal-cutting height, traction and cutting power, some of them are independent of one another, while most of them are correlated. For example, there is little relevance between a shearer's cutting depth and its cutting height, while there is great correlation between traction velocity and traction power. Thus, we will conduct independent analysis for attributes with little or no relevance to simplify the process and co relational analysis for attributes with strong correlation to avoid major discrepancies from the actual weight changes. According to the above-mentioned order $p_{1}^{\prime}, p_{2}^{\prime}, \ldots p_{m}^{\prime}$ of design plans, $\mathrm{R}$ is, as a result, modified as follows:

$$
R^{\prime}=\left[\begin{array}{cccc}
r_{11}^{\prime} & r_{12}^{\prime} & \cdots & r_{1 n}^{\prime} \\
r_{21}^{\prime} & r_{22}^{\prime} & \cdots & r_{2 n}^{\prime} \\
\vdots & \vdots & \ddots & \vdots \\
r_{m 1}^{\prime} & r_{m 2}^{\prime} & \cdots & r_{m n}^{\prime}
\end{array}\right]=\left[\begin{array}{l}
r_{1}^{\prime} \\
r_{2}^{\prime} \\
\vdots \\
r_{m}^{\prime}
\end{array}\right] \text {, from which we can }
$$

get priority matrix of multiple attributes:

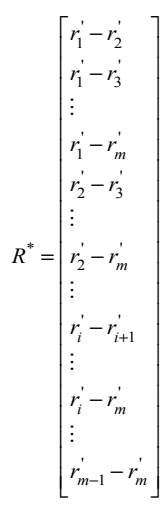


Table 1. Raw data of preliminary shearer design plans.

\begin{tabular}{|c|c|c|c|c|c|c|}
\hline Attribute & $\begin{array}{c}\text { Max Cutting Power } \\
\text { (kw) }\end{array}$ & $\begin{array}{c}\text { Max Traction Power } \\
\text { (kw) }\end{array}$ & $\begin{array}{l}\text { Max Traction } \\
\text { Velocity (kw) }\end{array}$ & $\begin{array}{l}\text { Max Cutting Depth } \\
(\mathbf{m m})\end{array}$ & Reliability & Sensitivity \\
\hline 1 & 750 & 110 & 21 & 865 & 6 & 9 \\
\hline 2 & 400 & 55 & 15 & 800 & 6 & 9 \\
\hline 3 & 1000 & 120 & 12.9 & 865 & 6 & 7 \\
\hline 4 & 650 & 110 & 20 & 800 & 7 & 5 \\
\hline 5 & 500 & 60 & 12.8 & 800 & 5 & 6 \\
\hline 6 & 800 & 20 & 22.6 & 865 & 5 & 8 \\
\hline
\end{tabular}

Satisfying the condition $R^{*} \bullet W^{T}>0$, in which, $W^{T}=\left(\omega_{1}, \omega_{2}, \cdots \omega_{n}\right)^{T}, \sum_{j=1}^{n} \omega_{j}=1 \omega_{j} \in[0,1]$.

when attributes of a shearer are independent of one another, the variation range of weight $\omega_{j}$ to keep the priority order unchanged is: $\bar{\omega}_{j}=\left[\bar{\omega}_{j \min }, \bar{\omega}_{j \max }\right], j=1,2 \cdots n$, whose restraint is: $\left\{\begin{array}{l}R^{*} \bullet W^{T}>0 \\ \sum_{i=1}^{n} \omega_{i}=1 \\ 0 \leq \omega_{i} \leq 1, i=1,2 \cdots n\end{array}\right.$

to facilitate parameter setting, relevance among shear attributes can be resolved to the following two categories:

(a) When two attributes maintain stable relevant priority, the ratio of their weights stays unchanged, i.e. $\frac{\omega_{i}}{\omega_{j}}=\frac{\omega_{i}^{\prime}}{\omega_{j}^{\prime}}=c_{1}$, in which $\omega_{i}, \omega_{j}$ are original attribute weights and $\omega_{i}^{\prime}, \omega_{j}{ }_{j}$ are attribute weights after the change in work conditions of the shear or the designer's idea, and $c_{1}=$ constant.

(b) Define the evaluation set of shear attributes as $Q=\left\{q_{1}, q_{2}, \cdots q_{n}\right\}$ and according to the features of relevant attributes in shear design, modify it to $Q=\left\{Q_{1}, Q_{2}, \cdots Q_{l}\right\}$, in which $Q_{l}$ is the $l^{\text {th }}$ subset of $Q$. If, after the change of the order of design plans, the weight of the $j$ th attribute in $Q_{l}$ meets $\sum_{Q_{l}} \omega_{j}=\sum_{Q_{l}} \omega_{j}{ }^{\prime}=c_{2}$, in which $\omega_{j}$ and $\omega_{j}{ }^{\prime}$ represent the modified attribute weights, and $c_{2}$ is a constant, then $c_{2}$ is the weight coefficient of the subset of $Q_{l}$.

When correlation among attributes is taken into account in weight sensitivity analysis, add the condition $\frac{\omega_{i}}{\omega_{j}}=$ $\frac{\omega_{i}^{\prime}}{\omega_{j}^{\prime}}=c_{1}, \sum_{Q_{l}} \omega_{j}=\sum_{Q_{l}} \omega_{j}{ }^{\prime}=c_{2}$ into restraint conditions of independent attributes and turn the restraint to

$$
\left\{\begin{array}{l}
R^{*} \bullet W^{T}>0 \\
\sum_{i=1}^{n} \omega_{i}=1 \\
0 \leq \omega_{i} \leq 1, i=1,2 \cdots n \\
\frac{\omega_{i}}{\omega_{j}}=c_{1} \\
\sum_{Q} \omega_{l}=c_{2}
\end{array}\right.
$$

In which, $Q$ is attribute evaluation set and $\omega_{l}$ is the attribute weight of the $l^{\text {th }}$ subset in attribute evaluation set $\mathrm{Q}$.

\section{CASE STUDY}

\subsection{Case 1}

Sort six preliminary shearer design plans according to their overall performance in six indicators as of max cutting power, max traction power, max traction velocity, max cutting depth, reliability and sensitivity (shown in Table 1), and identify the important attributes.

Step 1: From Table 1, we can obtain the following feature matrix A of evaluation indicators of six preliminary shearer design plans.

$$
A=\left[\begin{array}{llllll}
750 & 110 & 21 & 865 & 6 & 9 \\
400 & 55 & 15 & 800 & 6 & 9 \\
1000 & 120 & 12.9 & 865 & 6 & 7 \\
650 & 110 & 20 & 800 & 7 & 5 \\
500 & 60 & 12.8 & 800 & 5 & 6 \\
800 & 20 & 22.6 & 865 & 5 & 8
\end{array}\right]
$$


Step 2: Obtain comparison matrix E according to the correlation of max cutting power, max traction power, max traction velocity, max cutting depth, reliability and sensitivity.

$$
E=\left[\begin{array}{cccccc}
0 & 1 & -1 & 1 & -1 & -1 \\
-1 & 0 & -1 & -1 & -1 & -1 \\
1 & 1 & 0 & 0 & -1 & -1 \\
-1 & 1 & 0 & 0 & -1 & -1 \\
1 & 1 & 1 & 1 & 0 & -1 \\
1 & 1 & 1 & 1 & 1 & 0
\end{array}\right]
$$

Calculate attribute weight $\mathrm{W}$,

$W=[0.1218,0.0626,0.1439,0.1031,0.2373,0.3312]^{T}$

Step 3: Eliminate the discrepancies among different dimensions of attributes with standardized membership matrix $\mathrm{R}$ of feature matrix $\mathrm{A}$.

$$
R=\left[\begin{array}{cccccc}
0.5833 & 0.90 & 0.8367 & 1 & 0.5 & 1.00 \\
0.0000 & 0.35 & 0.2244 & 0 & 0.0 & 1.00 \\
1.0000 & 1.00 & 0.0102 & 1 & 1.0 & 0.50 \\
0.4167 & 0.90 & 0.7347 & 0 & 0.50 & 0.00 \\
0.1667 & 0.40 & 0.0000 & 0 & 0.75 & 0.25 \\
0.6667 & 0.00 & 1.0000 & 1 & 0.75 & 0.75
\end{array}\right]
$$

Work out the comprehensive evaluation values of preliminary shearer design plans

$R \bullet W=[0.8007,1.1121,0.5991,0.8385,0.3061,0.7546]^{T}$,

from which we can get the rank of comprehensive evaluation values of preliminary plans, i.e. $M_{2}>M_{4}>M_{1}>M_{6}>$ $M_{3}>M_{5}$. According to this, we can get

$R^{*}=\left[\begin{array}{cccccc}-0.5833 & -0.55 & -0.6123 & -1 & -0.5 & 0.00 \\ -1.0000 & -0.65 & 0.1224 & -1 & -1 & 0.50 \\ -0.4167 & -0.55 & -0.5103 & 0 & -0.5 & 1 \\ -0.1667 & -0.05 & 0.2244 & 0 & -0.75 & 0.75 \\ -0.6667 & 0.35 & -0.7756 & -1 & -0.75 & 0.25 \\ -0.1666 & 0 & -0.102 & -1 & 0 & -1 \\ -0.5833 & -0.1 & 0.7245 & -1 & -0.5 & -0.5 \\ 0.25 & 0.5 & 0.7347 & 0 & -0.25 & -0.25 \\ -0.25 & 0.9 & -0.2653 & -1 & -0.25 & -0.75 \\ -0.4167 & -0.1 & 0.8265 & 0 & -0.5 & 0.5 \\ 0.4166 & 0.5 & 0.8367 & 1 & -0.25 & -0.25 \\ -0.0834 & 0.9 & -0.1633 & 0 & -0.25 & -0.25 \\ -0.3333 & -1 & 0.9898 & 0 & -0.25 & 0.25 \\ 0.5 & -0.4 & 1 & 1 & 0 & 0.5 \\ 0.8333 & 0.6 & 0.0102 & 1 & 0.25 & 0.25\end{array}\right]$

Step 4: With attributes being uncorrelated, we can get the variation range of attribute weight that can maintain the priority order of design plans unchanged, as shown in Table 2.
Table 2. Weight range of uncorrelated attributes.

\begin{tabular}{|c|c|c|c|}
\hline & Attribute & Weight & Weight Range \\
\hline \hline 1 & Max Cutting Power & 0.1218 & $0-0.4023$ \\
\hline 2 & Max Traction Power & 0.0626 & $0-0.3333$ \\
\hline 3 & Max Traction Velocity & 0.1439 & $0-0.5548$ \\
\hline 4 & Max Cutting Depth & 0.1031 & $0-0.5048$ \\
\hline 5 & Reliability & 0.2373 & $0-0.5$ \\
\hline 6 & Sensitivity & 0.3312 & $0.1061-0.5$ \\
\hline
\end{tabular}

Table 3. Weight ranges of correlated attributes.

\begin{tabular}{|c|c|c|c|}
\hline & Attribute & Weight & Weight Range \\
\hline \hline 1 & Max Cutting Power & 0.1218 & $0-0.2412$ \\
\hline 2 & Max Traction Power & 0.0626 & $0.0623-0.33$ \\
\hline 3 & Max Traction Velocity & 0.1439 & $0-0.2677$ \\
\hline 4 & Max Cutting Depth & 0.1031 & $0-0.3031$ \\
\hline 5 & Reliability & 0.2373 & $0.1715-0.3350$ \\
\hline 6 & Sensitivity & 0.3312 & $0.1715-0.3350$ \\
\hline
\end{tabular}

It can be seen from the table that range lengths of various attributes vary from one another and the biggest range of max traction velocity indicates its weakest sensitivity and vice versa. Weight ranges of attribute 1 to 5 all start from 0 , while that of sensitivity cannot start from 0 , which indicates the priority of sensitivity is higher than that of other attributes, and therefore one should be discreet when it comes to value attribute 6 .

Step 5: analyze the correlation of attributes in shearer design: (1) traction power and traction velocity are closely related to each other, and thus categorize max traction power and max traction velocity into a subclass, with weight coefficient being $1 / 3$, and restraint condition being $\omega_{2}+\omega_{3}=1 / 3$; (2) a shearer's high reliability indicates that its parts and the whole machine are of longer failure-free operation duration and lower failure rate; high sensitivity indicates a shearer is of better mobility and maneuverability. If these two attributes are of equal priority, then the relative priority coefficient of reliability to sensitivity is 1 , restraint condition $\frac{\omega_{5}}{\omega_{6}}=1$, and the attribute weight ranges that keep the rank of design plans unchanged is as shown in Table 3.

It can be seen from Table $\mathbf{3}$ that attribute weight ranges have changed to maintain the rank of design plan unchanged. Ranges of reliability and sensitivity have the smallest change, which indicates they are the most sensitive; that the 
Table 4. Data Sheet of Preliminary Plans.

\begin{tabular}{|c|c|c|c|c|c|c|}
\hline Attribute & $\begin{array}{c}\text { Groove Depth } \\
(\mathbf{m m})\end{array}$ & $\begin{array}{c}\text { Groove-root Width } \\
(\mathbf{m m})\end{array}$ & $\begin{array}{c}\text { Coefficient of Pro- } \\
\text { cessing Difficulty }\end{array}$ & $\begin{array}{c}\text { Tensile Strength } \\
(\mathbf{M P a})\end{array}$ & Reliability & Fracture Sensitivity \\
\hline \hline 1 & 8 & 8 & 0.7 & 980 & 6 \\
\hline 2 & 8 & 16 & 0.4 & 980 & 7 & 5 \\
\hline 3 & 8 & 2 & 0.6 & 980 & 7 & 7 \\
\hline 4 & 8 & 16 & 0.4 & 885 & 5 & 7 \\
\hline 5 & 8 & 2 & 0.6 & 885 & 6 \\
\hline 6
\end{tabular}

Table 5. Independent attribute weight range.

\begin{tabular}{|c|c|c|c|}
\hline & Attribute & Weight & Weight Range \\
\hline \hline 1 & Groove Depth & 0.1312 & 0.4175 \\
\hline 2 & Groove-root Width & 0.0671 & $0-0.3134$ \\
\hline 3 & Coefficient of Processing Difficulty & 0.1457 & $0-0.5735$ \\
\hline 4 & Tensile Strength & 0.1101 & $0-0.5051$ \\
\hline 5 & Reliability & 0.2403 & $0-0.52$ \\
\hline 6 & Fracture Sensitivity & 0.3400 & $0.1102-0.510$ \\
\hline
\end{tabular}

beginning value of weight ranges of attribute 2 and 5 change from zero to a non-zero value which indicates the priority of max traction power, reliability and sensitivity is higher than that of other attributes. Thus, one should be discreet when it comes to fix values for these three attributes.

\subsection{Case 2}

\subsubsection{Experiment Plan and Data}

Take for example the torque-axis unload groove in the cutting unit of MG750/1800-WD shearer. The unload groove is designed to protect the transmission system and motor through instant fracture when overload happens. Sort the overall performance of six preliminary design plans based on six indicators (i.e. fracture torque value, groove-root width, coefficient of processing difficulty, material tensile strength, reliability and fracture sensitivity; some of them are shown in Table 4) of attributes of the torque-axis unload groove in the cutting unit of a shearer, and identify the important attributes. Groove 1 is a $40 \mathrm{Cr}$ - trapezoidal groove, Groove 2 a $40 \mathrm{Cr}$ - U-shaped groove, Groove 3 a 40Cr- V-shaped groove, Groove 4 a 20CrMo- U-shaped groove, Groove 5 a 20CrMo$\mathrm{V}$-shaped groove and Groove 6 a 20CrMo- trapezoidal groove.
We can find decision matrix A from Table 5.

$$
A=\left[\begin{array}{llllll}
8 & 8 & 0.7 & 980 & 6 & 9 \\
8 & 16 & 0.4 & 980 & 7 & 8 \\
8 & 2 & 0.6 & 980 & 5 & 6 \\
8 & 16 & 0.4 & 885 & 7 & 7 \\
8 & 2 & 0.6 & 885 & 5 & 9 \\
8 & 8 & 0.7 & 885 & 6 & 6
\end{array}\right]
$$

Through calculation, we can get weight ranges of independent attributes as shown in Table $\mathbf{5}$ and those of correlated attributes in Table 6.

It can be seen from Table $\mathbf{6}$ that attribute weight ranges have changed to maintain the rank of design plan unchanged. Ranges of reliability and sensitivity have the smallest change, which indicates they are the most sensitive; that the beginning value of weight ranges of attribute 1,4 and 5 change from zero to a non-zero value indicates the priority of groove depth, reliability and fracture sensitivity is higher than that of other attributes. Thus, the values of these three attributes should be discreetly chosen when feature matrix A is identified. 
Table 6. Weight ranges of correlated attributes.

\begin{tabular}{|c|c|c|c|}
\hline & Attribute & Weight & Weight Range \\
\hline \hline 1 & Groove Depth & 0.1312 & $0.06150-0.2430$ \\
\hline 2 & Groove-root Width & 0.0671 & $0-0.341$ \\
\hline 3 & Coefficient of Processing Difficulty & 0.1457 & $0-0.2702$ \\
\hline 4 & Tensile Strength & 0.1101 & $0.1045-0.3111$ \\
\hline 5 & Reliability & 0.2403 & $0.1697-0.3403$ \\
\hline 6 & Fracture Sensitivity & 0.3400 & $0.1697-0.3403$ \\
\hline
\end{tabular}

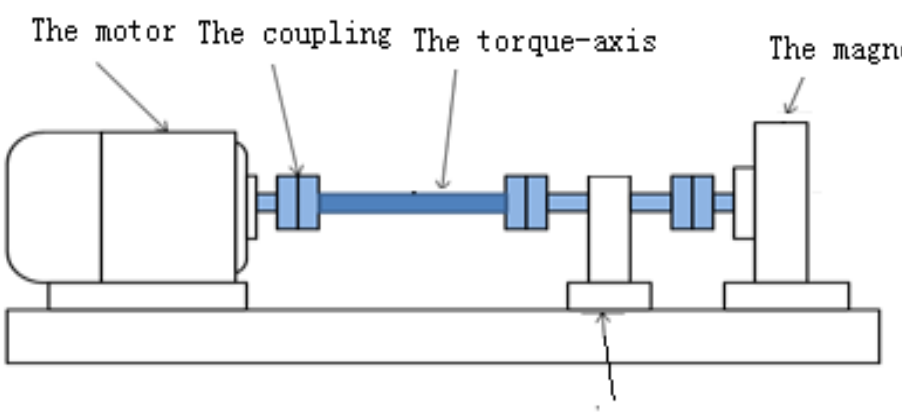

The torque sensor

Fig. (1). The test platform structure.

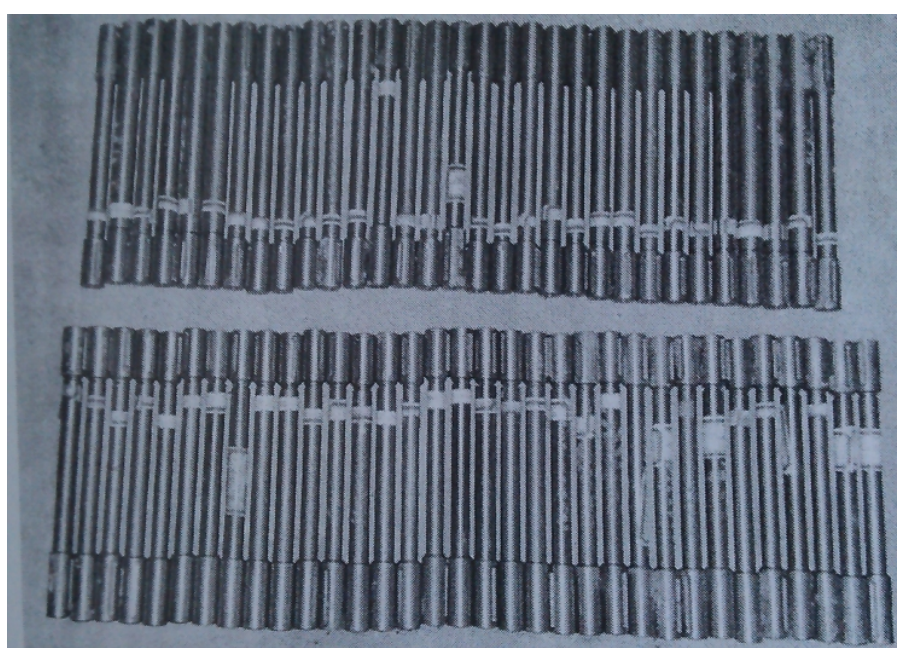

Fig. (2). A photo of test torque axis.

\subsubsection{Result Comparison}

We design a special test platform to verify the accuracy of the rank of preliminary torque-axis design plans obtained from the above-mentioned method. The test platform is made up of a motor, a frequency converter, a torque sensor, a magnetic particle brake and a magnetic particle brake controller, as shown in Fig. (1). The converter can slowly start the motor to reach the set resolution speed, the torque sensor can continuously collect torque and velocity signals and the magnetic brake can produce the set torque output through its controller. Torque axes used in the test will be made with the same material and in the same structure and diminished proportionally to $1 / 3$ of the sizes of the original axes. 10 test pieces will be processed for Plans 1,2,4 and 6;12 test pieces will be processed for Plans 3 and 5. As it is known from the table of preliminary plans that Plans 3 and 5 are of poor reliability, two spare test pieces are made (as shown in Fig. 2).

Test data are shown in Fig. (3), in which horizontal axis represents the number of test torque piece and vertical axis represents the multiplying rate of load at the moment of fracture. 1-10 represent test torque pieces of Plan 1 and they fracture under about 1.5 times of the rating load; $11-20$ rep- 


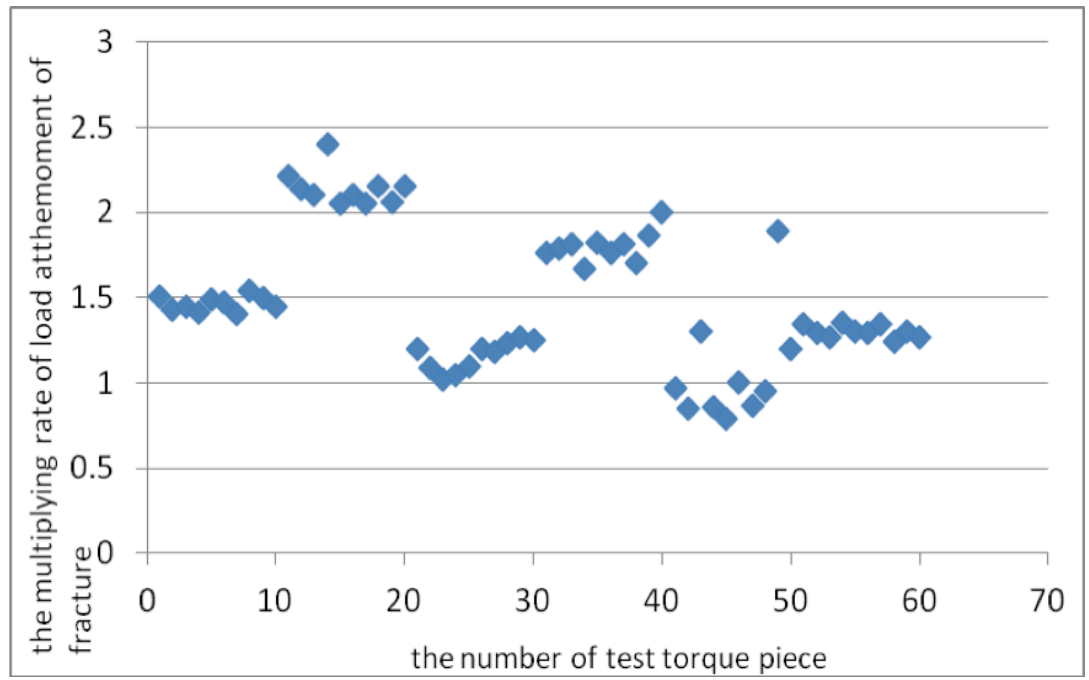

Fig. (3). Test data.

resent test torque pieces of Plan 2 and they fracture under about 2.2 times of the rating load; 21-30 represent test torque pieces of Plan 3 and they fracture under about 1.2 times of the rating load; 31-40 represent test torque pieces of Plan 4 and they fracture under about 1.75 times of the rating load; 41-50 represent test torque pieces of Plan 5 and they fracture under about 0.9 times of the rating load; 51-60 represent test torque pieces of Plan 6 and they fracture under about 1.4 times of the rating load. The plan order obtained from test results is basically in agreement with that from the abovementioned method.

\section{CONCLUSION}

In this paper, an algorithm to analyze the sensitivity of a shearer's multiple attributes is proposed to provide designers and decision-makers with quantified theoretical basis, in which the authors offer restraint conditions for independent and correlated attributes respectively, find out the attributes with higher sensitivity and thus obtain the attributes with relative higher priority.

\section{CONFLICT OF INTEREST}

The authors confirm that this article content has no conflict of interest.

\section{ACKNOWLEDGEMENTS}

The work presented in this paper was supported by the National Natural Science Foundation of China (Grants No.
51105266), Key Technology Special Subjects Foundation of Shanxi Province (No.20111101040), Youth Foundation of Taiyuan University of Technology (No.20132008).

\section{REFERENCES}

[1] Z.X. Sun, M. Han, W.H. Qiu, "Classification approach for multiple attribute decision making problem", Control Dec, vol. 21, no. 6, pp. 171-174, 2006.

[2] Y. Jiang, D.G. Tian, Y. Pan, "Ranking environmental projects model based on multicriteria decision-making and the weight sensitivity analysis", J. Syst. Eng. Electron., vol. 18, no. 3, pp. 534-539, 2007.

[3] J. Figueira, S. Greco, Y. Ehrgott, Multiple Criteria Decision Analysis: State of the Art Surveys. Kluwer Academic Publishers: Norwell M A, 2005

[4] H. Ding, Z. J. Yang, "Research and practice of shearer conceptual design fusion reasoning model", J. China Soc., vol. 35, no. 10, pp. 1748-1753, 2010.

[5] H. Ding, Z. J. Yang, "Method and system of shearer cutting unit modern design oriented to KBE", J. China Soc., vol. 37, no. 10, pp. 1765-1770, 2012.

[6] H. Ding, Z. J. Yang, and Y. Wang, "Research on digital design system for coal mining machine based on KBE", J. Mach. Des, no. 4, pp. 15-19, 2011.

[7] Q. Fu, and X.Y. Zhao, Principle and Application of Projection Pursuit Model. Science Press: Beijing, 2006.

[8] S. L. Chen, J. G. Li, and X. G. Wang, Fuzzy Sets Theory And applications. Science Press: Beijing, 2005.

[9] S. Q. Xu, Multi-objective Decision Analysis of Diversion Study of Divers Ion Standards Based on Entropy. Wuhan University: Wuhan, 2004.

[10] T. L. Saaty, "Multicriteria Decision Making: the Analytic Hierarchy Process: Planning, Priority Setting, Resourceal Location". $2^{\text {nd }}$ ed. Pittsburgh, Cal., RWS Publications: USA, 1980.

(C) Qiuxia and Zhaojian; Licensee Bentham Open.

This is an open access article licensed under the terms of the Creative Commons Attribution Non-Commercial License (http://creativecommons.org/licenses/ by-nc/4.0/) which permits unrestricted, non-commercial use, distribution and reproduction in any medium, provided the work is properly cited. 